\title{
ON THE STRUCTURE OF $S$ AND $C(S)$ FOR $S$ DYADIC
}

BY

\section{JAMES HAGLER}

ABSTRACT. A dyadic space $S$ is defined to be a continuous image of $\{0,1\}^{m}$ for some infinite cardinal number $m$. We deduce Banach space properties of $C(S)$ and topological properties of $S$. For example, under certain cardinality restrictions on $m$, we show: Every dyadic space of topological weight m contains a closed subset homeomorphic to $\{0,1\}^{\mathrm{m}}$. Every Banach space $X$ isomorphic to an $m$ dimensional subspace of $C(S)$ (for $S$ dyadic) contains a subspace isomorphic to $l^{1}(\Gamma)$ where $\Gamma$ has cardinality $m$.

Introduction. In this paper we examine the relationships between dyadic spaces $S$ and the Banach spaces $C(S)$ of continuous real valued functions on $S$. The main result is

THEOREM 1. Let $S$ be a dyadic space and $m$ a regular cardinal number. Then the following are equivalent:

(i) The topological weight of $S \geqslant m$.

(ii) $C(S)$ contains a subspace isomorphic to $l_{m}^{1}$.

(iii) $C(S)^{*}$ contains a subspace isometrically isomorphic to $L^{1}\{0,1\}^{m}$.

(iv) If $\phi: D^{\mathfrak{n}} \rightarrow S$ is continuous and onto, then there exists a closed subset $\Omega$ of $D^{\mathfrak{n}}$, homeomorphic to $D^{m}$, such that $\left.\phi\right|_{\Omega}$ is a homeomorphism.

(v) $C(S)$ contains a subspace isometrically isomorphic to $C\left(D^{\mathrm{m}}\right)$ which is the range of a projection in $C(S)$ of norm one.

(A cardinal number $m$ is regular if $m$ is not the sum of fewer than $m$ cardinal numbers each smaller than $m$. For any cardinal number $n, D^{n}=\{0,1\}^{n}$ is a generalized Cantor set. A dyadic space is a compact Hausdorff space which is a continuous image of some $D^{\mathfrak{n}}$. For the definitions of the other terms, see $\S 1$ below.)

Properties of $C(S)$ spaces for $S$ dyadic (especially questions about their isomorphic classification) have previously been examined by Pelczyński [12] in his monograph. Combining his Proposition 8.4 with our Theorem 1, we have the following

Presented to the Society, May 2, 1974; received by the editors June 3, 1974 and, in revised form, December 15, 1974. 54 F65.

AMS (MOS) subject classifications (1970). Primary 46E15, 46B99; Secondary 54A25, 
COROLLARY: Let $\mathrm{m}$ be a regular cardinal number and $S$ a dyadic space of topological weight $\mathrm{m}$.

Then $C(S)$ is isomorphic to $C\left(D^{m}\right)$ if $S$ satisfies any one of the following properties:

(1) There exists a continuous onto map $\phi: D^{n} \rightarrow S$ (for some $\left.n\right)$ admitting an averaging operator.

(2) If $S$ is homeomorphic to a closed subset of a compact Hausdorff space $K$, then there exists a linear extension operator $E: C(S) \rightarrow C(K)$.

(3) $S$ is a closed subset of some $D^{\mathfrak{n}}$ and there is a retraction $r: D^{\mathfrak{n}} \rightarrow S$.

(For the definitions of averaging and extension operators and retraction, see [12].)

Topological properties of dyadic spaces somewhat weaker than (i) $\Rightarrow$ (iv) of Theorem 1 are known and due to Efimov [2]. In particular, he proved (under certain restrictions on $m$ ) that if $S$ is dyadic and has topological weight $m$, then $D^{\mathrm{m}}$ is a continuous image of a closed subset of $S$.

Using Theorem 1 in an obvious fashion, we can deduce a dyadic space analogue of a well-known result of Kuratowski [6, p. 444] on uncountable compact metric spaces.

Corollary. Let $S_{1}$ and $S_{2}$ be dyadic spaces and $m$ the topological weight of $S_{2}$. Assume that $m$ is a regular cardinal.

Then if $\phi: S_{1} \rightarrow S_{2}$ is continuous and onto, there exists a closed subset $\Omega$ of $S_{1}$, homeomorphic to $D^{\mathfrak{m}}$, such that $\left.\phi\right|_{\Omega}$ is a homeomorphism.

We now indicate the organization of this paper. $\$ 1$ contains definitions and notation. In $\$ 2$ we investigate the structure of nonseparable subspaces $X$ of $C(S)$ for $S$ dyadic. For example, in Theorem 2 we prove that if $X$ is an $m$ dimensional subspace of $C(S)$ (for $S$ dyadic; again, $m$ is a regular cardinal), then $X$ contains a subspace isomorphic to $l_{m}$. This yields immediately a partial affirmative answer to a conjecture of Rosenthal [14] concerning conjugate $L^{1}(\mu)$ spaces.

$\$ 3$ contains the proof of Theorem 1. The only difficult implication is (iii) $\Rightarrow$ (iv). ((i) $\Rightarrow$ (ii) is immediate from Theorem 2 and the other implications follow easily from known results.) The major step in the proof of (iii) $\Rightarrow$ (iv) is Proposition 11, which gives a sufficient condition for pairs of sets $\left\{\left(A_{\alpha}, B_{\alpha}\right)\right.$ : $\alpha \in A\}$ in $D^{\mathfrak{n}}$ to contain a "large" independent subfamily $\left\{\left(A_{\alpha}, B_{\alpha}\right): \alpha \in B\right\}$ where $B \subset A$.

The proofs of our results use a combinatorial lemma of Erdös-Radó (cf. [3] for an easy proof as well as for a coherent presentation of many of the known results on dyadic spaces.) We state this result as Lemma 7. In addition, we require some further combinatorial results, especially a "Ramsey-like" theorem 
for functions (Lemma 8). To establish properties of subspaces of $C(S)$ for $S$ dyadic, we rely heavily on a result of Rosenthal [15, Proposition 4] which gives sufficient conditions for a set of functions on some set $S$ to be equivalent (in sup norm) to the usual basis of $l_{\mathrm{m}}^{1}$. We state this as Lemma 6 .

1. Preliminaries. For the most part our notation and terminology is standard. All Banach spaces will be real Banach spaces and all operators between two Banach spaces will be bounded linear operators. We will denote the dual space of the Banach space $X$ by $X^{*}$. Two Banach spaces $X$ and $Y$ are isomorphic if there is a linear homeomorphism from $X$ onto $Y$, isometrically isomorphic if there is a surjective operator $T: X \rightarrow Y$ such that $\|T x\|=\|x\|$ for every $x \in X$.

Let $\Gamma$ be a set. Then $l^{1}(\Gamma)\left(l^{\infty}(\Gamma)\right.$ respectively) is the Banach space of real valued functions $f: \Gamma \rightarrow \mathbf{R}$ such that $\|f\|=\Sigma\{|f(\gamma)|: \gamma \in \Gamma\}<\infty(\|f\|=$ $\sup \{|f(\gamma)|: \gamma \in \Gamma\}<\infty$ respectively). If $\operatorname{card}(\Gamma)=\mathfrak{m}>\aleph_{0}$, then we denote $l^{1}(\Gamma)$ by $l_{\mathrm{m}}^{1}$ and $l^{\infty}(\Gamma)$ by $l_{\mathrm{m}}^{\infty}$. (Of course, card $(\Gamma)$ denotes the cardinality of the set $\Gamma$.) If $\operatorname{card}(\Gamma)=\aleph_{0}$, then we denote $l^{1}(\Gamma)$ by $l^{1}$ and $l^{\infty}(\Gamma)$ by $l^{\infty}$.

Let $\Omega$ be a set, $\Sigma$ a $\sigma$-algebra of subsets of $\Omega$, and $\mu$ a (positive) measure. Then by $L^{1}(\mu)=L^{1}(\Omega, \Sigma, \mu)$ we mean the Banach space of equivalence classes of $\mu$-measurable functions such that $\|f\|=\int|f| d \mu<\infty$. If $\Omega=\{0,1\}^{m}, \Sigma$ is the $\sigma$-algebra of Borel sets in $\Omega$, and $\mu$ is normalized Haar measure on the topological group $\{0,1\}^{\mathrm{m}}$, we denote $L^{1}(\Omega, \Sigma, \mu)$ by $L^{1}\{0,1\}^{\mathrm{m}}$. (Another way of viewing $L^{1}\{0,1\}^{m}$ is as follows: Let $M$ be a set of cardinality $m$, and for each $m \in M$, define a measure $\mu_{m}$ on $\{0,1\}$ by $\mu_{m}\{0\}=\not 2=\mu_{m}\{1\}$. If $\mu$ is the product measure of the $\mu_{m}$ 's on $\{0,1\}^{M}$, then $L^{1}(\mu)$ is isometric to $L^{1}\{0,1\}^{m}$.)

Let $M$ be a set of cardinality $m$. By $D^{\mathrm{m}}$ we mean the generalized Cantor set $\{0,1\}^{M}$. We denote a point in $\{0,1\}^{M}$ by $\xi=\left(\xi_{m}\right)_{m \in M}$. If $M$ is a subset of $M$, then $P_{M}(\xi)=\left(\xi_{m}\right)_{m \in M} \in\{0,1\}^{M}$ is the projection of $\{0,1\}^{M}$ onto its factor $\{0,1\}^{M}$.

A function $f:\{0,1\}^{M} \rightarrow \mathbf{R}$ is said to depend on the coordinates in $M(M \subset$ $M)$ (or more simply, $f$ depends on $M$ ) if there is a function $g:\{0,1\}^{M} \rightarrow \mathbf{R}$ such that $f(\xi)=g\left(P_{M}(\xi)\right)$ for all $\xi \in\{0,1\}^{M}$.

Let $A$ be an index set, and $\left\{\left(A_{\alpha}, B_{\alpha}\right): \alpha \in A\right\}$ a family of pairs of subsets of some set $\Gamma$ with $A_{\alpha} \cap B_{\alpha}=\varnothing$ for all $\alpha$. Then (following Rosenthal [15]) we say that $\left\{\left(A_{\alpha}, B_{\alpha}\right): \alpha \in A\right\}$ is independent if for every pair of disjoint finite subsets $P$ and $N$ of $A,\left(\bigcap_{\alpha \in P} A_{\alpha}\right) \cap\left(\bigcap_{\alpha \in N} B_{\alpha}\right) \neq \varnothing$. Also, we say that $\left\{\left(A_{\alpha}, B_{\alpha}\right)\right.$ : $\alpha \in A\}$ is 2-independent if for every pair of distinct $\alpha, \beta \in A, A_{\alpha} \cap A_{\beta} \neq \varnothing, A_{\alpha}$ $\cap B_{\beta} \neq \varnothing, B_{\alpha} \cap A_{\beta} \neq \varnothing$ and $B_{\alpha} \cap B_{\beta} \neq \varnothing$.

If $S$ is a topological space, then the topological weight of $S$ (denoted wt( $(S)$ ) is the least cardinal number $m$ such that there exists a base of neighborhoods in $S$ of 
cardinality $m$. The dimension of a Banach space $X$ (denoted $\operatorname{dim}(X)$ ) is the least cardinal number $m$ such that there exists a set in $X$ of cardinality $m$ whose closed linear span is all of $X$.

The author wishes to thank the referee, and B. Hoffmann and R. Haydon for pointing out errors in early versions of the manuscript.

2. Subspaces of $C(S)$ for $S$ dyadic. It is well known (cf. [1]) that any separable Banach space is isometric to a subspace of $C\left(D^{\aleph_{0}}\right)$, hence of $C\left(D^{\mathfrak{n}}\right)$ for any infinite cardinal number $n$. Theorem 2 shows that $C\left(D^{\mathfrak{n}}\right)$ is not "rich" in nonseparable subspaces.

THEOREM 2. Let $\mathrm{m}$ be a regular cardinal number. Let $X$ be a Banach space of dimension $m$ which is isomorphic to a subspace of $C\left(D^{\eta}\right)$ for some $n$. Then $X$ contains a subspace isomorphic to $l_{\mathrm{m}}^{1}$.

Before giving the proof we consider some consequences.

COROLlARY 3. Let $\mathrm{m}$ be a regular cardinal number, $S$ a dyadic space, and $X$ a subspace of $C(S)$ of dimension $m$. Then $X$ contains a subspace isomorphic to $l_{\mathrm{m}}^{1}$.

Proof. If $S$ is dyadic, then there is a continuous onto map $\phi: D^{n} \rightarrow S$ for some $n$. As is well known, $\phi$ induces an isometric embedding $\phi^{0}: C(S) \rightarrow C\left(D^{n}\right)$ defined by $\phi^{0} f(\xi)=f(\phi(\xi))$ for $f \in C(S), \xi \in D^{n}$. So the first part follows from Theorem 2. The second part also follows easily since $\operatorname{dim} C(S)=w t(S)$ for any compact Hausdorff space $S$. Q.E.D.

REMARKS. (1) The conclusion of Corollary 3 (hence of Theorem 2) is false if $m$ is the sum of a countable number of smaller cardinals. Indeed, in [12, Example 7, p. 71], Pelczyński shows that in this case there exists a dyadic space $S$ of weight $m$ such that $C(S)$ does not contain a subspace isomorphic to $l_{\mathrm{m}}^{1}$. We do not know if either Theorem 1 or Theorem 2 holds if we assume only that $m$ is not the sum of a countable number of smaller cardinals.

(2) Other possible improvements of Theorem 2 appear to be false. For example, it is not true that every nonseparable subspace of $L^{\infty}\{0,1\}^{N_{0}}$ contains a subspace isomorphic to $\boldsymbol{l}^{\mathbf{1}}$. For by a result of R. C. James [7], [9], there exists a separable Banach space $X$ with $X^{*}$ nonseparable but such that neither $X$ nor any successive dual of $X$ contains a subspace isomorphic to $l^{1}$. But since the dual of every separable space is isomorphic to a subspace of $L^{\infty}\{0,1\}^{\aleph_{0}}$, there is an isomorph of $X^{*}$ in $L^{\infty}\{0,1\}^{N_{0}}$ which does not contain $l^{1}$.

Implication (ii) $\Rightarrow$ (iv) of the next corollary gives an affirmative answer in special cases to a conjecture of Pelczyński [13].

Corollary 4. Let $X$ be a Banach space and $S$ a dyadic space with $X$ isomorphic to a subspace of $C(S)$. Let $\mathrm{m}$ be a regular cardinal number. Then 
the following are equivalent:

(i) The dimension of $X \geqslant m$.

(ii) $X^{*}$ contains d subspace isomorphic to $L^{1}\{0,1\}^{\mathbf{m}}$.

(iii) $X^{*}$ contains a subspace isomorphic to $C\left(D^{\mathrm{m}}\right)^{*}$.

(iv) $X$ contains a subspace isomorphic to $l_{\mathrm{m}}^{1}$.

Proof. (i) $\Rightarrow$ (iv) is Corollary 3 , (iv) $\Rightarrow$ (iii) follows from [13, Proposition 3.3] or [5, Lemma 2], (iii) $\Rightarrow$ (ii) is trivial, and (ii) $\Rightarrow$ (i) follows from this observation: It is easy and well known that if $R$ is a reflexive subspace of $X^{*}$, then $R^{*}$ is isomorphic to a quotient space of $X$. Since $L^{1}\{0,1\}^{\mathbf{m}}$ contains a subspace isomorphic to $L^{2}\{0,1\}^{m}$, it follows (assuming (ii)) that $L^{2}\{0,1\}^{m}$ is isomorphic to a quotient space of $X$. Since $\operatorname{dim} L^{2}\{0,1\}^{\mathrm{m}}=\mathrm{m}$, we must have $\operatorname{dim} X \geqslant \mathrm{~m}$, which establishes (i). Q.E.D.

REMARK. Implication (ii) $\Rightarrow$ (iv) may hold if we assume only that $m$ is an infinite cardinal number.

As a final corollary to Theorem 2, we can characterize a large number of $L^{1}(\mu)$ spaces isomorphic to conjugate spaces of subspaces of $C(S)$ for $S$ dyadic.

Corollary 5. Let $\mathrm{m}$ be a regular cardinal number. Let $X$ be a closed subspace of $C\left(D^{\text {mI }}\right)$ of dimension $m$.

If $X^{*}$ is isomorphic to $L^{1}(\mu)$ for some measure $\mu$, then $X^{*}$ is isomorphic to $C\left(D^{\mathfrak{m}}\right)^{*}$.

Proof. Since $X$ contains a subspace isomorphic to $l_{\mathbf{m}}^{\mathbf{l}}$, this follows immediately from [14, Remark 5, p. 242]. Q.E.D.

We now begin to assemble the components necessary in the proof of Theorem 2. Crucial to our proof is the following result of Rosenthal [15, Proposition 4] which gives sufficient conditions for a set of functions to be equivalent (in sup norm) to the usual basis of $l_{\mathrm{m}}^{1}$.

LEMMA 6. Let $m \geqslant \aleph_{0}$ and let $A$ be a set of cardinality $m$. Let $r$ and $\delta$ be real numbers with $\delta>0$. Let $\left\{f_{\alpha}: \alpha \in A\right\}$ be norm one functions in $l^{\infty}(\Gamma)$, and put

$$
A_{\alpha}=\left\{\gamma: f_{\alpha}(\gamma) \geqslant r+\delta\right\}, \quad B_{\alpha}=\left\{\gamma: f_{\alpha}(\gamma) \leqslant r\right\} .
$$

If $\left\{\left(A_{\alpha}, B_{\alpha}\right): \alpha \in A\right\}$ is an independent family of pairs of subsets of $\Gamma$, then $\left\{f_{\alpha}\right.$ : $\alpha \in A\}$ is $\delta / 2$ equivalent to the usual basis of $l_{\mathrm{m}}^{1}$. More precisely, given distinct $\alpha_{1}, \ldots, \alpha_{k} \in A$ and scalars $t_{1}, \ldots, t_{k}$, then

$$
\frac{\delta}{2} \sum_{i=1}^{k}\left|t_{i}\right| \leqslant\left\|\sum_{i=1}^{k} t_{i} f_{\alpha_{i}}\right\| \leqslant \sum_{i=1}^{k}\left|t_{i}\right| .
$$

To obtain such functions, we shall begin with a family of functions satisfy- 
ing weaker properties than those of Lemma 6 . Then we shall use some combinatorial results to extract a subfamily of these functions (of the desired cardinality) satisfying the lemma. The first of these combinatorial lemmas is due to Erdös and Radó (cf. [3] for an easy proof).

LEMMA 7. Let $m$ be a regular cardinal number. Let $A$ be a set of cardinality $m$, and for each $\alpha \in A$, let $N_{\alpha}$ be a finite subset of some set $\Gamma$. Then there exists a subset $A^{\prime} \subset A$ with $\operatorname{card}\left(A^{\prime}\right)=m$ such that $N_{\alpha_{1}} \cap N_{\alpha_{2}}=\bigcap_{\alpha \in A^{\prime}} N_{\alpha}$ for every pair of distinct $\alpha_{1}, \alpha_{2} \in A^{\prime}$.

The other combinatorial result we need is a "Ramsey-like" theorem for functions.

Lemma 8. Let $\theta>0$, let $m$ be an infinite cardinal number, and let $A$ be $a$ set of cardinality $m$. Let $\left\{f_{\alpha}: \alpha \in A\right\}$ be functions in $l^{\infty}(\Gamma)$ such that $\left\|f_{\alpha}-f_{\beta}\right\|$ $\geqslant \theta$ for every pair of distinct $\alpha, \beta \in A$.

If $\Gamma=\Gamma_{1} \cup \cdots \cup \Gamma_{n}$, then there exists an $i$ and a subset $A^{\prime} \subset A$ with $\operatorname{card}\left(A^{\prime}\right)=m$ such that $\left\|\left.f_{\alpha}\right|_{\Gamma_{i}}-\left.f_{\beta}\right|_{\Gamma_{i}}\right\| \geqslant \theta / 2$ for every pair of distinct $\alpha, \beta \in A^{\prime}$.

Proof. If $m=\aleph_{0}$, then the lemma follows directly from Ramsey's theorem (cf. [10]). So let us assume that $m>\aleph_{0}$. We present the proof in the case of $n=2$. The general case follows easily either by induction or by an obvious elaboration (with more notation) of the argument for $n=2$.

Let us say that a subset $A$ of $A$ works on $\Gamma_{i}$ if $\left\|\left.f_{\alpha}\right|_{\Gamma_{i}}-\left.f_{\beta}\right|_{i}\right\| \geqslant \theta / 2$ for every pair of distinct $\alpha, \beta \in A$.

Let $F=\left\{\left(A_{1}, A_{2}\right): A_{i}\right.$ works on $\Gamma_{i}$ for $\left.i=1,2\right\}$ partially ordered by $\left(A_{1}, A_{2}\right) \leqslant\left(B_{1}, B_{2}\right)$ if $A_{1} \subset B_{1}$ and $A_{2} \subset B_{2}$. Observe that $F \neq \varnothing$, and that if $\left(A_{1}^{t}, A_{2}^{t}\right)_{t \in T}$ is a linearly ordered set in $F$, then $\left(\mathrm{U}_{t} A_{1}^{t}, \mathrm{U}_{t} A_{2}^{t}\right)$ is an upper bound in $F$.

By Zorn's lemma there exists a maximal element $\left(A_{1}, A_{2}\right)$ in $F$. We claim that either $i=1$ and $A^{\prime}=A_{1}$ or $i=2$ and $A^{\prime}=A_{2}$ satisfies the conclusion of the lemma. We assume that neither does and derive a contradiction.

If both card $\left(A_{1}\right)$ and card $\left(A_{2}\right)<m$, then, putting $B=A \sim\left(A_{1} \cup A_{2}\right)$, $\operatorname{card}(B)=m$. Since $\left(A_{1}, A_{2}\right)$ is maximal in $F$, for every $\beta \in B$ there exists an $\alpha_{1} \in A_{1}$ and $\alpha_{2} \in A_{2}$ such that

$$
\left\|f_{\alpha_{i}}\left|\Gamma_{i}-f_{\beta}\right|_{\Gamma_{i}}\right\|<\theta / 2 \text { for } i=1,2 .
$$

Define $\Psi: B \rightarrow A_{1} \times A_{2}$ by $\Psi(\beta)=\left(\alpha_{1}, \alpha_{2}\right)$, where $\alpha_{1} \in A_{1}$ and $\alpha_{2} \in A_{2}$ are chosen to have (*) for $\beta$. Since $\operatorname{card}(B)>\operatorname{card}\left(A_{1} \times A_{2}\right), \Psi$ cannot be one-toone. Thus, for some $\left(\alpha_{1}, \alpha_{2}\right) \in A_{1} \times A_{2}$, there exist distinct $\beta, \sigma \in B$ such that $\beta, \sigma \in \Psi^{-1}\left(\alpha_{1}, \alpha_{2}\right)$. But then 


$$
\begin{aligned}
\left\|f_{\beta}-f_{\sigma}\right\| & =\max _{i=1,2}\left\|\left.f_{\beta}\right|_{\Gamma_{i}}-\left.f_{\sigma}\right|_{\Gamma_{i}}\right\| \\
& \leqslant \max _{i=1,2}\left\|\left.f_{\beta}\right|_{\Gamma_{i}}-f_{\alpha_{i}}\left|\Gamma_{i}\|+\| f_{\alpha_{i}}\right| \Gamma_{i}-\left.f_{\sigma}\right|_{\Gamma_{i}}\right\| \\
& <\theta / 2+\theta / 2=\theta
\end{aligned}
$$

by the triangle inequality. This contradicts the assumption that both $\operatorname{card}\left(A_{1}\right)$ $<m$ and $\operatorname{card}\left(A_{2}\right)<m$, and the proof is complete. Q.E.D.

The final preliminary result is this easy lemma.

LEMma 9. Let $0<\theta<1$, let $\mathrm{m}$ be a cardinal number which is not the sum of a countable number of smaller cardinals, and let $A$ be a set of cardinality $m$. Let $\left\{f_{\alpha}: \alpha \in A\right\}$ be norm one functions in $l^{\infty}(\Gamma)$ such that $\left\|f_{\alpha}-f_{\beta}\right\| \geqslant \theta$ for every pair of distinct $\alpha, \beta \in A$. Then there exists a rational number $r \in[-1,1]$ and a subset $B \subset A$ with $\operatorname{card}(B)=m$ such that

$$
A_{\alpha}=\left\{\gamma: f_{\alpha}(\gamma) \geqslant r+\theta / 4\right\} \neq \varnothing, \quad B_{\alpha}=\left\{\gamma: f_{\alpha}(\gamma) \leqslant r\right\} \neq \varnothing
$$

for each $\alpha \in B$.

Proof. For each rational number $r \in[-1,1]$, put $A_{r}=\{\alpha \in A: \alpha$ satisfies (*) for $r$. We are done once we show that $\operatorname{card}\left(U_{r} A_{r}\right)=m$. For then, by the assumptions on $m, \operatorname{card}\left(A_{r}\right)=m$ for some $r$, and we may take $B=A_{r}$.

If $\operatorname{card}\left(\bigcup_{r} A_{r}\right)<m$, then the set $A^{\prime}=A \sim \bigcup_{r} A_{r}$ has cardinality $m$ and for each $\alpha \in A^{\prime}$, $\sup _{\gamma} f_{\alpha}(\gamma)-\inf _{\gamma} f_{\alpha}(\gamma)<\theta / 2$. Since $\operatorname{card}\left(A^{\prime}\right)>\aleph_{0}$, we can easily find a rational number $r$ and distinct $\alpha_{1}, \alpha_{2} \in A^{\prime}$ such that inf $f_{\alpha_{i}}(\gamma)<$ $r<\sup _{\gamma} f_{\alpha_{i}}(\gamma)$ for $i=1,2$. Let $1_{r}$ denote the function on $\Gamma$ whose constant value is $r$. Then $\left\|f_{\alpha_{1}}-f_{\alpha_{2}}\right\| \leqslant\left\|f_{\alpha_{1}}-1_{r}\right\|+\left\|1_{r}-f_{\alpha_{2}}\right\|<\theta$ which is a contradiction. Q.E.D.

We now turn to the proof of Theorem 2. Since the property of containing $l_{\mathrm{m}}^{1}$ is invariant under isomorphism, we may assume that $X$ is an m dimensional subspace of $C\left(D^{\mathfrak{n}}\right)$. Thus, for any $0<\theta<1$, there exists a set $A$ of cardinality $m$ and a set $\left\{x_{\alpha}: \alpha \in A\right\}$ of norm one elements in $X$ such that $\left\|x_{\alpha}-x_{\beta}\right\| \geqslant \theta$ for distinct $\alpha, \beta \in A$. Theorem 2 is immediate from

Proposition 10. Let $17 / 18<\theta<1$, m a regular cardinal number, and A a set of cardinality m. Let $\left\{x_{\alpha}: \alpha \in A\right\}$ be a set of norm one elements in $C\left(D^{n}\right)$ such that $\left\|x_{\alpha}-x_{\beta}\right\| \geqslant \theta$ for distinct $\alpha, \beta \in A$.

Then there exists a subset $B \subset A$ of cardinality m such that $\left\{x_{\alpha}: \alpha \in B\right\}$ is equivalent to the usual basis of $l_{\mathfrak{m}}^{1}$.

Proof. Choose $0<\epsilon<1 / 36$. Let $N$ be a set of cardinality $n$; we regard $D^{n}=\{0,1\}^{N}$. Let $F$ be the algebra of functions in $C\{0,1\}^{N}$ which depend on a 
finite set of coordinates in $N$. Then $F$ separates the points of $\{0,1\}^{N}$ and contains the constant functions. By the Stone-Weierstrass theorem, there exists for each $\alpha \in A$ a norm one function $f_{\alpha} \in$ Fand a finite subset $N_{\alpha} \subset N$ such that $\left\|f_{\alpha}-x_{\alpha}\right\|$ $<\epsilon$ and $f_{\alpha}$ depends on $N_{\alpha}$. Thus, for a distinct $\alpha, \beta \in A$ we have $\left\|f_{\alpha}-f_{\beta}\right\| \geqslant$ $\theta-2 \epsilon$.

By Lemma 7 there exists $A^{\prime} \subset A$ with $\operatorname{card}\left(A^{\prime}\right)=m$ and a finite (perhaps empty) subset $N \subset N$ such that $N=\bigcap_{\alpha \in A^{\prime}} N_{\alpha}=N_{\alpha} \cap N_{\beta}$ for every pair of distinct $\alpha, \beta \in A^{\prime}$.

The proof is somewhat different from this point depending on whether or not $N=\varnothing$. As the proof in the case $N \neq \varnothing$ is more complicated, we present it in detail, then indicate how to handle the case $N=\varnothing$.

If $N \neq \varnothing$, write $N=\left\{n_{1}, \ldots, n_{k}\right\}$. Let $e_{1}, \ldots, e_{2^{k}}$ be an enumeration of the $2^{k}$ points in $\{0,1\}^{N}$ and define

$$
\Gamma_{i}=\left\{\xi \in\{0,1\}^{N}: P_{N}(\xi)=e_{i}\right\}
$$

for $1 \leqslant i \leqslant 2^{k}$. This partitions $\{0,1\}^{N}$ into $2^{k}$ disjoint closed open sets $\Gamma_{1}$, $\ldots, \Gamma_{2} k$. By Lemma 8 there exists an $i$ and a subset $A^{\prime \prime} \subset A^{\prime}$ with $\operatorname{card}\left(A^{\prime \prime}\right)$ $=m$ such that $\left\|\left.f_{\alpha}\right|_{\Gamma_{i}}-\left.f_{\beta}\right|_{\Gamma_{i}}\right\| \geqslant(\theta-2 \epsilon) / 2$ for every pair of distinct $\alpha, \beta \in A^{\prime \prime}$. By Lemma 9 there exists a rational number $r$ and a subset $B \subset A^{\prime \prime}$ with $\operatorname{card}(B)$ $=\mathfrak{m}$ such that both

$$
A_{\alpha}^{i}=\left\{\gamma \in \Gamma_{i}: f_{\alpha}(\gamma) \geqslant r+(\theta-2 \epsilon) / 8\right\} \text { and } B_{\alpha}^{i}=\left\{\gamma \in \Gamma_{i}: f_{\alpha}(\gamma) \leqslant r\right\}
$$

are nonempty for every $\alpha \in B$.

Since $\Gamma_{i}=\left\{e_{i}\right\} \times\{0,1\}^{N \sim N}$, each of the sets $A_{\alpha}^{i}$ and $B_{\alpha}^{i}$ depend on the coordinates $N_{\alpha} \sim N$ in $\Gamma_{i}$. Hence if $\alpha, \beta \in B$ are distinct, then $A_{\alpha}^{i}$ and $B_{\alpha}^{i}$ depend on coordinates (in $\Gamma_{i}$ ) disjoint from those on which $A_{\beta}^{i}$ and $B_{\beta}^{i}$ depend. From this it is immediate that the family $\left\{\left(A_{\alpha}^{i}, B_{\alpha}^{i}\right): \alpha \in B\right\}$ is independent in $\Gamma_{i}$. Indeed, let distinct $\alpha_{1}, \ldots, \alpha_{m}, \alpha_{m+1}, \ldots, \alpha_{q}$ be given. For $1 \leqslant j \leqslant m$, pick $a_{j} \in\{0,1\}^{N_{\alpha_{j}} \sim N}$ such that if $\gamma \in \Gamma_{i}$ and $\left.P_{N_{\alpha j} \sim N} \sim \gamma\right)=a_{j}$, then $\gamma \in A_{\alpha_{j}}^{i}$. Similarly for $m+1 \leqslant j \leqslant q$, pick $b_{j} \in\{0,1\}^{N_{\alpha j} \sim N}$ such that if $\gamma \in \Gamma_{i}$ and $P_{N_{\alpha_{j}} \sim N}(\gamma)=b_{j}$, then $\gamma \in B_{\beta_{j}}^{i}$. Define $\gamma \in \Gamma_{i}$ by

and

$$
\boldsymbol{P}_{\boldsymbol{N}_{\alpha_{j}} \sim N}(\gamma)= \begin{cases}a_{j} & \text { if } 1 \leqslant j \leqslant m, \\ b_{j} & \text { if } m+1 \leqslant j \leqslant q,\end{cases}
$$

$$
P_{n}(\gamma)=0 \quad \text { if } n \notin N \cup\left(\bigcup_{\alpha \in B} N_{\alpha} \sim N\right) .
$$

Then $\gamma \in\left(\bigcap_{j=1}^{m} A_{\alpha_{j}}^{i}\right) \cap\left(\bigcap_{j=m+1}^{q} B_{\beta_{j}}^{i}\right)$ as required.

Now for each $\alpha \in B$, put 
$A_{\alpha}=\left\{\xi \in\{0,1\}^{N}: f_{\alpha}(\xi) \geqslant r+(\theta-2 \epsilon) / 8\right\}, \quad B_{\alpha}=\left\{\xi \in\{0,1\}^{N}: f_{\alpha}(\xi) \leqslant r\right\}$.

Since $A_{\alpha} \cap B_{\alpha}=\varnothing$, and $A_{\alpha} \supset A_{\alpha}^{i}$ and $B_{\alpha} \supset B_{\alpha}^{i}$ for all $\alpha \in B$, we have that $\left\{\left(A_{\alpha}, B_{\alpha}\right): \alpha \in B\right\}$ is an independent family of pairs of sets in $\{0,1\}^{\mathrm{N}}$. Since $\left\|f_{\alpha}\right\|=1$ for all $\alpha \in B$, it follows from Lemma 6 that $\left\{f_{\alpha}: \alpha \in B\right\}$ is $(\theta-2 \epsilon) / 16$ equivalent to the usual basis of $l_{\mathfrak{m}}^{1}$.

(If $N=\varnothing$, we can apply Lemma 9 immediately to the set $\left\{f_{\alpha}: \alpha \in \mathrm{A}^{\prime}\right\}$ and proceed identically as above to obtain the set $B$ and the independent family of pairs of sets $\left\{\left(A_{\alpha}, B_{\alpha}\right): \alpha \in B\right\}$.)

Finally, the following standard perturbation argument shows that $\left\{x_{\alpha}: \alpha \in\right.$ $B\}$ is $(\theta-2 \epsilon) / 16-\epsilon=(\theta-18 \epsilon) / 16>1 / 32$ equivalent to the usual basis of $l_{m}^{1}$. Let $q$, distinct $\alpha_{1}, \ldots, \alpha_{q} \in B$, and scalars $t_{1}, \ldots, t_{q}$ be given. Then

$$
\begin{aligned}
\left\|\sum_{j=1}^{q} t_{j} x_{\alpha_{j}}\right\| & \geqslant\left\|\sum_{j=1}^{q} t_{j} f_{\alpha_{j}}\right\|-\sum_{j=1}^{q}\left|t_{j}\right||| f_{\alpha_{j}}-x_{\alpha_{j}}|| \\
& \geqslant \frac{\theta-2 \epsilon}{16} \sum_{j=1}^{q}\left|t_{j}\right|-\epsilon \sum_{j=1}^{q}\left|t_{j}\right| \geqslant \frac{1}{32} \sum_{j=1}^{q}\left|t_{j}\right| ;
\end{aligned}
$$

also, since $\left\|x_{\alpha_{j}}\right\|=1$ for each $j,\left\|\Sigma_{j=1}^{q} t_{j} x_{\alpha_{j}}\right\| \leqslant \Sigma_{j=1}^{q}\left|t_{j}\right|$. This completes the proof of Proposition 10, hence of Theorem 2. Q.E.D.

3. The structure of dyadic spaces. This section contains the proof of Theorem 1 . We begin by giving sufficient conditions for a family $\left\{\left(A_{\alpha}, B_{\alpha}\right)\right.$ : $\alpha \in A\}$ of pairs of subsets of $D^{\mathfrak{n}}$ to contain a "large" independent subfamily $\left\{\left(A_{\alpha}, B_{\alpha}\right): \alpha \in B\right\}$. As above, we regard $D^{n}=\{0,1\}^{N}$ for some set $N$ of cardinality $n$.

PROPOSITION 11. Let $\mathrm{m}$ be a regular cardinal number and let $\mathrm{A}$ be a set of cardinality $\mathrm{m}$.

Assume that $\left\{\left(A_{\alpha}, B_{\alpha}\right): \alpha \in \mathrm{A}\right\}$ is a 2-independent family of pairs of closed open subsets of $\{0,1\}$. Then there exist

(1) a subset $B \subset A$ with $\operatorname{card}(B)=m$;

(2) a finite subset $N \subset N$, an element $e \in\{0,1\}^{N}$ and a closed open subset $\Gamma \subset\{0,1\}^{N}$ defined by

$$
\Gamma=\left\{\xi \in\{0,1\}^{N}: P_{N}(\xi)=e\right\}=\{e\} \times\{0,1\}^{N \sim N} ;
$$

and

(3) disjoint finite subsets $N_{\alpha} \subset N \sim N($ for $\alpha \in B)$ so that $A_{\alpha} \cap \Gamma$ and $B_{\alpha} \cap \Gamma$ are both nonempty and depend in $\Gamma$ only on the coordinates $N_{\alpha}$.

In particular, $\left\{\left(A_{\alpha}, B_{\alpha}\right): \alpha \in B\right\}$ is an independent family of pairs of sets.

Proof. For each $\alpha \in A$, define $f_{\alpha} \in C\{0,1\}^{\mathrm{N}}$ by 


$$
f_{\alpha}(\xi)=\left\{\begin{aligned}
1 & \text { if } \xi \in A_{\alpha} \\
-1 & \text { if } \xi \in B_{\alpha} \\
0 & \text { otherwise }
\end{aligned}\right.
$$

Since every closed open subset of $\{0,1\}^{N}$ depends on a finite set of coordinates in $N$, each function $f_{\alpha}$ depends on some finite subset $M_{\alpha} \subset N$.

By Lemma 7 there exists a subset $A^{\prime} \subset A$ with $\operatorname{card}\left(A^{\prime}\right)=\mathfrak{m}$ and a finite (perhaps empty) subset $N \subset N$ such that $N=\bigcap_{\alpha \in A^{\prime}} M_{\alpha}=M_{\alpha} \cap M_{\beta}$ for every pair of distinct $\alpha, \beta \in A^{\prime}$. If $N=\varnothing$ then it follows immediately (cf. the proof of Proposition 10) that $B=A^{\prime}, N=\varnothing, \Gamma=\{0,1\}^{N}$ and $N_{\alpha}=M_{\alpha}$ for $\alpha \in B$ satisfy (1)-(3) of the proposition.

If $N \neq \varnothing$, write $N=\left\{n_{1}, \ldots, n_{k}\right\}$. Let $e_{1}, \ldots, e_{2 k}$ be an enumeration of the $2^{k}$ points in $\{0,1\}^{N}$, and define

$$
\Gamma_{i}=\left\{\xi \in\{0,1\}^{N}: P_{N}(\xi)=e_{i}\right\}
$$

for $1 \leqslant i \leqslant 2^{k}$. For fixed $i,\left.f_{\alpha}\right|_{\Gamma_{i}}$ depends on the coordinates $N_{\alpha}=M_{\alpha} \sim N$. Hence, if $\alpha, \beta \in A^{\prime}$ are distinct, $\left.f_{\alpha}\right|_{\Gamma_{i}}$ and $\left.f_{\beta}\right|_{\Gamma_{i}}$ depend on disjoint sets of coordinates. Thus, given $\gamma_{\alpha} \in \Gamma_{i}$ for each $\alpha \in A^{\prime}$, there exists a $\gamma \in \Gamma_{i}$ such that

$$
f_{\alpha}(\gamma)=f_{\alpha}\left(\gamma_{\alpha}\right) \text { for each } \alpha \in A^{\prime} \text {. }
$$

To see this, define $\gamma \in \Gamma_{i}$ by $P_{N_{\alpha}}(\gamma)=P_{N_{\alpha}}\left(\gamma_{\alpha}\right)$ for $\alpha \in A^{\prime}, P_{N}(\gamma)=e_{i}$ and $P_{n}(\gamma)$ $=0$ if $n \notin\left(\cup_{\alpha \in A^{\prime}} N_{\alpha}\right) \cup N$. This $\gamma$ clearly satisfies (*).

To finish the proof, it suffices to show that for some $1 \leqslant i \leqslant 2^{k}$, there exists a subset $B \subset A^{\prime}$ with $\operatorname{card}(B)=\mathrm{m}$ such that

$$
\text { for each } \alpha \in B, f_{\alpha}\left(\Gamma_{i}\right) \supset\{-1,1\} \text {. }
$$

For once we have such a set $B$, then we take $\Gamma=\Gamma_{i}$ and we are done. To see that $\left\{\left(A_{\alpha}, B_{\alpha}\right): \alpha \in B\right\}$ is an independent family, let distinct $\alpha_{1}, \ldots, \alpha_{r}, \beta_{1}$, $\ldots, \beta_{s} \in B$ be given. Combining (*) and (**), we obtain a $\gamma \in \Gamma_{i}$ such that

$$
f_{\alpha_{j}}(\gamma)=1 \quad \text { for } 1 \leqslant j \leqslant r, \quad f_{\beta_{q}}(\gamma)=-1 \quad \text { for } 1 \leqslant q \leqslant s .
$$

But this is equivalent to

$$
\gamma \in\left(\bigcap_{j=1}^{r} A_{\alpha_{j}}\right) \cap\left(\bigcap_{q=1}^{s} B_{\beta_{q}}\right)
$$

which establishes the independence of $\left\{\left(A_{\alpha}, B_{\alpha}\right): \alpha \in B\right\}$.

So it remains to find $i$ and the set $B$ satisfying (**) for this $i$. For each $1 \leqslant i \leqslant 2^{k}$, define

$$
A_{i}^{\prime}=\left\{\alpha \in A^{\prime}: f_{\alpha}\left(\Gamma_{i}\right) \supset\{1,-1\}\right\}
$$


If some $A_{i}^{\prime}$ has cardinality $m$, then we put $B=A_{i}^{\prime}$ and we are done. So let us assume that $\operatorname{card}\left(A_{i}^{\prime}\right)<m$ for each $i$ and derive a contradiction.

Put $C=A^{\prime} \sim \bigcup_{i=1}^{2 k} A_{i}^{\prime}$. Then $\operatorname{card}(C)=\mathfrak{m}$, and for $n o \beta \in C$ does $f_{\beta}$ satisfy (**) for any $i$. Thus, the map $\Psi: C \rightarrow\{-1,0,1\}^{2^{k}}$ defined by

$$
\Psi(\beta)_{i}=\left\{\begin{aligned}
1 & \text { if } 1 \in f_{\beta}\left(\Gamma_{i}\right), \\
-1 & \text { if }-1 \in f_{\beta}\left(\Gamma_{i}\right), \\
0 & \text { if }\left.f_{\beta}\right|_{\Gamma_{i}}=0
\end{aligned}\right.
$$

is well defined.

Since $\operatorname{card}(C)=m$ and $\operatorname{card}\{-1,0,1\} 2^{k}=3^{2^{k}}$, there exist distinct $\alpha, \beta \in$ C such that $\Psi(\alpha)=\Psi(\beta)$. But this implies that $\left\|f_{\alpha}-f_{\beta}\right\| \leqslant 1$, since $f_{\alpha}$ and $f_{\beta}$ never differ by 2 on any $\Gamma_{i}$. This is a contradiction, since the assumption of 2 independence of $\left\{\left(A_{\alpha}, B_{\alpha}\right): \alpha \in A\right\}$ implies that $\left\|f_{\alpha}-f_{\beta}\right\|=2$ for distinct $\alpha, \beta$ $\in$ A. Q.E.D.

We turn now to the proofs of the equivalences in Theorem 1, giving the easier implications first, and leaving the most difficult one ((iii) $\Rightarrow$ (iv)) until the end.

Proof of (i) $\Rightarrow$ (ii). It is well known that $\operatorname{wt}(S)=\operatorname{dim} C(S)$ for any compact Hausdorff space $S$. By (i) $\Rightarrow$ (iv) of Corollary $4, C(S)$ contains a subspace isomorphic to $l_{m}^{1}$.

Proof of (ii) $\Rightarrow$ (iii). By (ii) $\Rightarrow$ (iii) of Corollary $4, C(S)^{*}$ contains a subspace isomorphic to $L^{1}\{0,1\}^{\mathrm{m}}$. But for any measure $\mu$, if $C(S)^{*}$ contains a subspace isomorphic to $L^{1}(\mu), C(S)^{*}$ contains a subspace isometrically isomorphic to $L^{1}(\mu)$. (This follows easily from Maharam's theorem [11].) Thus, $C(S)^{*}$ contains a subspace isometrically isomorphic to $L^{1}\{0,1\}^{\mathrm{m}}$.

Proof of (iv) $\Rightarrow$ (v). By Theorem 6.6 of [12], if $S$ is a compact Hausdorff space containing a homeomorph of $D^{\mathrm{m}}$, then $C(S)$ contains a subspace isometrically isomorphic to $C\left(D^{\mathrm{m}}\right)$ which is the range of projection in $C(S)$ of norm one.

Proof of (v) $\Rightarrow(\mathrm{i})$. If $C(S)$ contains a subspace isomorphic to $C\left(D^{\mathrm{m}}\right)$, then $\operatorname{dim} C(S) \geqslant \mathfrak{m}$. Hence $w t(S) \geqslant \mathfrak{m}$.

Proof OF (iii) $\Rightarrow$ (iv). Let $Z$ be a subspace of $C(S)^{*}$ isometric to $L^{1}\{0,1\}^{\mathrm{m}}$. By Lemma 1.3 of [14] there exists a probability measure $\mu \in C(S)^{*}$ such that $Z$ is a subspace of $L^{1}(\mu)$. It now follows easily from Maharam [11, Theorem 2] that there exists a sub- $\sigma$-algebra $\Sigma^{\prime}$ of the $\sigma$-algebra of Borel sets on $S$ such that $L^{1}\left(S, \Sigma^{\prime},\left.\mu\right|_{\Sigma^{\prime}}\right)$ is isometric to $L^{1}\{0,1\}^{n}$ for some $n \geqslant m$.

By Maharam's theorem [11], we may select a set $A$ of cardinality $m$ and measurable sets $\left\{O_{\alpha}: \alpha \in A\right\}$ in $\Sigma^{\prime}$ which satisfy the following property:

Given nonnegative integers $k$ and $q$ and distinct $\alpha_{1}, \ldots, \alpha_{k}, \beta_{1}, \ldots, \beta_{q} \in$ $A$, then 


$$
\mu\left(\bigcap_{i=1}^{k} o_{\alpha_{i}} \cap \bigcap_{j=1}^{q} \sim O_{\beta_{j}}\right)=2^{-(k+q)}
$$

Let $0<\epsilon<1 / 16$ and fix (for the moment) $\alpha \in A$. Define $r_{\alpha} \in L^{1}(\mu)$ by $r_{\alpha}=$ $2 \chi_{0_{\alpha}}-1$. (We regard $L^{1}(\mu)$ as a subspace of $C(S)^{*} ; \chi_{0}$ denotes the characteristic function of the measurable set 0 .) Since $\left\|r_{\alpha}\right\|=1$, there exists $g_{\alpha} \in C(S),\left\|g_{\alpha}\right\|$ $=1$, such that $\int g_{\alpha} r_{\alpha} d \mu \geqslant 1-\epsilon$.

Put $F_{\alpha}=\left\{s \in S: g_{\alpha}(s) \geqslant \epsilon\right\}$ and $G_{\alpha}=\left\{s \in S: g_{\alpha}(s) \leqslant-\epsilon\right\}$. We claim that $\mu\left(F_{\alpha}\right) \geqslant \mu\left(F_{\alpha} \cap O_{\alpha}\right) \geqslant 1 / 2-2 \epsilon>3 / 8$ and $\mu\left(G_{\alpha}\right) \geqslant \mu\left(G_{\alpha} \cap \sim O_{\alpha}\right) \geqslant 1 / 2-2 \epsilon>$ 3/8. We show this for $F_{\alpha}$, the proof for $G_{\alpha}$ being the same. If the assertion is false, then since $r_{\alpha}=1$ on $O_{\alpha}$ and $\mu\left(O_{\alpha}\right)=1 / 2$,

$$
\begin{aligned}
1 / 2-\epsilon & <\int_{0_{\alpha}} g_{\alpha} d \mu=\int_{0_{\alpha} \cap F_{\alpha}} g_{\alpha} d \mu+\int_{0_{\alpha} \sim F_{\alpha}} g_{\alpha} d \mu \\
& \leqslant \mu\left(0_{\alpha} \cap F_{\alpha}\right)+\epsilon / 2 \leqslant 1 / 2-2 \epsilon+\epsilon / 2 \leqslant 1 / 2-3 \epsilon / 2,
\end{aligned}
$$

a contradiction.

Having now selected $g_{\alpha}, F_{\alpha}$ and $G_{\alpha}$ as above for each $\alpha \in A$, we have easily that $\left\{\left(F_{\alpha}, G_{\alpha}\right): \alpha \in A\right\}$ is a 2 -independent family of closed sets in $S$. For given distinct $\alpha, \beta \in A$ and (for example) $F_{\alpha}$ and $F_{\beta}$, then $F_{\alpha} \cap O_{\alpha}$ and $F_{\beta} \cap O_{\beta}$ are sets each of measure $>3 / 8$ contained in a set of measure $3 / 4$ (namely $O_{\alpha} \cup$ $\left.0_{\beta}\right)$. It is clear from this that $\mu\left(F_{\alpha} \cap O_{\alpha} \cap F_{\beta} \cap O_{\beta}\right)>0$, whence $F_{\alpha} \cap F_{\beta} \neq \varnothing$. (The demonstrations that $F_{\alpha} \cap G_{\beta} \neq \varnothing, G_{\alpha} \cap F_{\beta} \neq \varnothing$, and $G_{\alpha} \cap G_{\beta} \neq \varnothing$ are identical to the above proof.) Finally, put $\widetilde{F}_{\alpha}=\left\{s: g_{\alpha}(s)>\epsilon / 2\right\}$ and $\widetilde{G}_{\alpha}=\{s$ : $\left.g_{\alpha}(s)<-\epsilon / 2\right\}$. Then $\widetilde{F}_{\alpha}$ and $\widetilde{G}_{\alpha}$ are disjoint open sets containing $F_{\alpha}$ and $G_{\alpha}$ respectively.

Now, let $\phi:\{0,1\}^{N} \rightarrow S$ be a continuous onto map, where $N$ is a set of cardinality $n$. For fixed $\alpha, \phi^{-1}\left(\widetilde{F}_{\alpha}\right)$ is an open set in $\{0,1\}^{N}$, so we may write $\phi^{-1}\left(\tilde{F}_{\alpha}\right)=U_{\gamma} U_{\gamma}$, where each $U_{\gamma}$ is closed and open in $\{0,1\}^{N}$. But then $\cup_{\gamma} U_{\gamma} \supset \phi^{-1}\left(F_{\alpha}\right)$ (which is compact), so there exist a finite number $\gamma_{1}, \ldots$, $\gamma_{r}$ of $\gamma$ 's such that

$$
\phi^{-1}\left(F_{\alpha}\right) \subset \bigcup_{j=1}^{r} U_{\gamma_{j}} \subset \phi^{-1}\left(\tilde{F}_{\alpha}\right) \text {. }
$$

Put $A_{\alpha}=\bigcup_{j=1}^{r} U_{\gamma_{j}}$. Then $A_{\alpha}$ is a closed open set in $\{0,1\}^{N}$. Similarly, we can find a closed open set $B_{\alpha}$ in $\{0,1\}^{N}$ such that

$$
\phi^{-1}\left(G_{\alpha}\right) \subset B_{\alpha} \subset \phi^{-1}\left(\tilde{G}_{\alpha}\right)
$$

Since $\phi\left(A_{\alpha}\right) \supset F_{\alpha}, \phi\left(B_{\alpha}\right) \supset G_{\alpha}$ and $A_{\alpha} \cap B_{\alpha}=\varnothing$ for each $\alpha$, it is clear that $\left\{\left(A_{\alpha}, B_{\alpha}\right): \alpha \in A\right\}$ is a 2-independent family of closed open sets in $\{0,1\}^{\mathrm{N}}$.

By Proposition 11, we can select the following:

(1) a subset $B \subset A$ with $\operatorname{card}(B)=m$; 
(2) a finite set $N \subset N$, an element $e \in\{0,1\}^{N}$ and a closed open subset $\Gamma \subset\{0,1\}^{N}$ defined by $\Gamma=\left\{\xi \in\{0,1\}^{N}: P_{N}(\xi)=e\right\}=\{e\} \times\{0,1\}^{N \sim N}$; and

(3) disjoint finite subsets $N_{\alpha} \subset N \sim N$ (for $\alpha \in B$ ) so that $A_{\alpha} \cap \Gamma$ and $B_{\alpha}$ $\cap \Gamma$ are both nonempty and depend in $\Gamma$ only on the coordinates $N_{\alpha}$.

From (3) it is clear that $\left\{\left(A_{\alpha} \cap \Gamma, B_{\alpha} \cap \Gamma\right): \alpha \in B\right\}$ is independent. For each $\alpha \in B$, pick $a_{\alpha}$ and $b_{\alpha} \in P_{N_{\alpha}}(\Gamma)$ such that if $\xi \in \Gamma$ and $P_{N_{\alpha}}(\xi)=a_{\alpha}$, then $\xi \in A_{\alpha} \cap \Gamma$, and if $P_{N_{\alpha}}(\xi)=b_{\alpha}$, then $\xi \in B_{\alpha} \cap \Gamma$. We define a subset $\Omega$ of $\Gamma$ as follows:

$$
\begin{aligned}
\Omega=\{\xi \in \Gamma: & P_{N_{\alpha}}(\xi)=a_{\alpha} \text { or } b_{\alpha} \text { for each } \alpha \in B, \\
& \text { and } \left.P_{n}(\xi)=0 \text { if } n \notin N \cup\left(\bigcup_{\alpha \in B} N_{\alpha}\right)\right\} .
\end{aligned}
$$

Since $D^{m}$ is homeomorphic to $\{0,1\}^{B}$, we can define a map $\sigma:\{0,1\}^{B} \rightarrow$ $\Omega$ by

$$
P_{N_{\alpha}}(\sigma(\xi))= \begin{cases}a_{\alpha} & \text { if } \xi_{\alpha}=0 \\ b_{\alpha} & \text { if } \xi_{\alpha}=1\end{cases}
$$

for all $\alpha \in B$. It is easy to see that $\sigma$ is a homeomorphism onto $\Omega$, so $\Omega$ is homeomorphic to $D^{\mathrm{m}}$.

To complete the proof it remains to show that $\left.\phi\right|_{\Omega}$ is one to one (hence, a homeomorphism on $\Omega$ ). Let distinct $\xi_{1}, \xi_{2} \in \Omega$ be given. Then for some $\alpha \in$ $B, P_{N_{\alpha}}\left(\xi_{1}\right) \neq P_{N_{\alpha}}\left(\xi_{2}\right)$. Without loss of generality, we can assume that $\xi_{1} \in A_{\alpha}$ and $\xi_{2} \in B_{\alpha}$. But $\phi\left(A_{\alpha}\right) \subset \widetilde{F}_{\alpha}$ and $\phi\left(B_{\alpha}\right) \subset \widetilde{G}_{\alpha}$, and since $\widetilde{F}_{\alpha} \cap \widetilde{G}_{\alpha}=\varnothing$, we have $\phi\left(\xi_{1}\right) \neq \phi\left(\xi_{2}\right)$. This completes the proof.

\section{REFERENCES}

1. S. Banach, Théorie des opérations linéaries, Monografie Mat., PWN, Warsaw, 1932; reprint, Chelsea, New York, 1955. MR 17, 175.

2. B. V. Efimov, Subspaces of dyadic bicompacta, Dokl. Akad. Nauk SSSR 185 (1969), 987-990 = Soviet Math. Dokl. 10 (1969), 453-456. MR 39 \#3459.

3. B. V. Efimov and R. Engelking, Remarks on dyadic spaces. II, Colloq. Math. 13 (1964/65), 181-197. MR 32 \#6391.

4. R. Engelking, Cartesian products and dyadic spaces, Fund. Math. 57 (1965), 287304. MR 33 \#4879.

5. J. Hagler and C. Stegall, Banach spaces whose duals contain complemented subspaces isomorphic to $C[0,1]^{*}$, J. Functional Analysis 13 (1973), 233-251.

6. P. R. Halmos, Measure theory, Von Nostrand, Princeton, N.J., 1950. MR 11, 504.

7. R. C. James, A separable somewhat reflexive Banach space with nonseparable dual, Bull. Amer. Math. Soc. 80 (1974), 738-743.

8. K. Kuratowski, Topology. Vol. 1, 5th ed., PWN, Warsaw; Academic Press, New York, 1966. MR 36 \#840.

9. J. Lindenstrauss and C. Stegall, Examples of separable spaces which do not contain $l_{1}$ and whose duals are non separable (preprint). N. Y., 1972.

10. C. L. Liu, Topics in combinatorial mathematics, Math. Assoc. of Amer., Buffalo, 
11. D. Maharam, On homogeneous measure algebras, Proc. Nat. Acad. Sci. U.S.A. 28 (1942), 108-111. MR 4, 12.

12. A. Pelczyński, Linear extensions, linear averagings, and their applications to linear topological classification of spaces of continuous functions, Dissertationes Mat. Rozprawy Mat. 58 (1968). MR 37 \#3335.

13. - On Banach spaces containing $L_{1}(\mu)$, Studia Math. 30 (1968), 231-246. MR 38 \#521.

14. H. P. Rosenthal, On injective Banach spaces and the spaces $L^{\infty}(\mu)$ for finite measures $\mu$, Acta Math. 124 (1970), 205-248. MR 41 \#2370.

15. A characterization of Banach spaces containing $l^{l}$, Proc. Nat. Acad. Sci. U. S. A. 71 (1974), 2411-2413.

DEPARTMENT OF MATHEMATICS, CATHOLIC UNIVERSITY OF AMERICA, WASHINGTON, D. C. 20064 